\title{
Some generalizations of operator inequalities for positive linear maps
}

Jianming $\mathrm{Xue}^{1^{*}}$ and Xingkai Hu${ }^{2}$

"Correspondence:

xuejianming104@163.com

'Oxbridge College, Kunming

University of Science and

Technology, Kunming, Yunnan 650106, P.R. China

Full list of author information is

available at the end of the article

\begin{abstract}
In this paper, we generalize some operator inequalities for positive linear maps due to Lin (Stud. Math. 215:187-194, 2013) and Zhang (Banach J. Math. Anal. 9:166-172, 2015).
\end{abstract}

MSC: 47A63;47A30

Keywords: operator inequalities; Kantorovich inequalities; positive linear maps

\section{Introduction}

Throughout this paper, let $M, M^{\prime}, m, m^{\prime}$ be scalars, $I$ be the identity operator, and $\mathcal{B}(\mathcal{H})$ be the set of all bounded linear operators on a Hilbert space $(\mathcal{H},\langle\cdot, \cdot\rangle)$. The operator norm is denoted by $\|\cdot\|$. We write $A \geq 0$ if the operator $A$ is positive. If $A-B \geq 0$, then we say that $A \geq B$. For $A, B>0$, we use the following notation:

$A \nabla_{\mu} B=(1-\mu) A+\mu B, A \sharp_{\mu} B=A^{\frac{1}{2}}\left(A^{-\frac{1}{2}} B A^{-\frac{1}{2}}\right)^{\mu} A^{\frac{1}{2}}$, where $0 \leq \mu \leq 1$.

When $\mu=\frac{1}{2}$ we write $A \nabla B$ and $A \sharp B$ for brevity for $A \nabla_{\frac{1}{2}} B$ and $A \sharp_{\frac{1}{2}} B$, respectively; see Kubo and Ando [3].

A linear map $\Phi$ is positive if $\Phi(A) \geq 0$ whenever $A \geq 0$. It is said to be unital if $\Phi(I)=I$. We say that $\Phi$ is 2-positive if whenever the $2 \times 2$ operator matrix $\left[\begin{array}{cc}A & B \\ B^{*} & C\end{array}\right]$ is positive, then so is $\left[\begin{array}{cc}\Phi(A) & \Phi(B) \\ \Phi\left(B^{*}\right) & \Phi(C)\end{array}\right]$.

Let $0<m \leq A, B \leq M$ and $\Phi$ be positive unital linear map. Lin [1], Theorem 2.1, proved the following reversed operator AM-GM inequalities:

$$
\begin{aligned}
& \Phi^{2}\left(\frac{A+B}{2}\right) \leq K^{2}(h) \Phi^{2}(A \sharp B), \\
& \Phi^{2}\left(\frac{A+B}{2}\right) \leq K^{2}(h)(\Phi(A) \sharp \Phi(B))^{2},
\end{aligned}
$$

where $K(h)=\frac{(h+1)^{2}}{4 h}$ with $h=\frac{M}{m}$ is the Kantorovich constant.

Can the inequalities (1.1) and (1.2) be improved? Lin [1], Conjecture 4.2, conjectured that the constant $K(h)$ can be replaced by the Specht ratio $S(h)=\frac{\frac{1}{h-1}}{e \log h^{\frac{1}{h-1}}}$ in (1.1) and (1.2), which remains as an open question.

Zhang [2], Theorem 2.6, generalized (1.1) and (1.2) when $p \geq 2$ :

$$
\Phi^{2 p}\left(\frac{A+B}{2}\right) \leq \frac{\left(K\left(M^{2}+m^{2}\right)\right)^{2 p}}{16 M^{2 p} m^{2 p}} \Phi^{2 p}(A \sharp B),
$$

(c) 2016 Xue and Hu. This article is distributed under the terms of the Creative Commons Attribution 4.0 International License (http://creativecommons.org/licenses/by/4.0/), which permits unrestricted use, distribution, and reproduction in any medium, provided you give appropriate credit to the original author(s) and the source, provide a link to the Creative Commons license, and indicate if changes were made. 


$$
\Phi^{2 p}\left(\frac{A+B}{2}\right) \leq \frac{\left(K\left(M^{2}+m^{2}\right)\right)^{2 p}}{16 M^{2 p} m^{2 p}}(\Phi(A) \sharp \Phi(B))^{2 p} .
$$

We will present some operator inequalities which are generalizations of (1.1), (1.2), (1.3), and (1.4) in the next section.

Bhatia and Davis [4] proved that if $0<m \leq A \leq M$ and $X$ and $Y$ are two partial isometries on $\mathcal{H}$ whose final spaces are orthogonal to each other. Then for every 2-positive unital linear map $\Phi$,

$$
\Phi\left(X^{*} A Y\right) \Phi\left(Y^{*} A Y\right)^{-1} \Phi\left(Y^{*} A X\right) \leq\left(\frac{M-m}{M+m}\right)^{2} \Phi\left(X^{*} A X\right) .
$$

Lin [5], Conjecture 3.4, conjectured that the following inequality could be true:

$$
\left\|\Phi\left(X^{*} A Y\right) \Phi\left(Y^{*} A Y\right)^{-1} \Phi\left(Y^{*} A X\right) \Phi\left(X^{*} A X\right)^{-1}\right\| \leq\left(\frac{M-m}{M+m}\right)^{2} .
$$

Recently, Fu and He [6], Theorem 5, proved

$$
\left\|\Phi\left(X^{*} A Y\right) \Phi\left(Y^{*} A Y\right)^{-1} \Phi\left(Y^{*} A X\right) \Phi\left(X^{*} A X\right)^{-1}\right\| \leq \frac{1}{4}\left(\left(\frac{M-m}{M+m}\right)^{2} M+\frac{1}{m}\right)^{2} .
$$

We will get a stronger result than (1.6).

\section{Main results}

We begin this section with the following lemmas.

Lemma 1 [7] Let $A, B>0$. Then the following norm inequality holds:

$$
\|A B\| \leq \frac{1}{4}\|A+B\|^{2} .
$$

Lemma 2 [8] Let $A>0$. Then for every positive unital linear map $\Phi$,

$$
\Phi\left(A^{-1}\right) \geq \Phi^{-1}(A)
$$

Lemma 3 [9] Let $A, B>0$. Then, for $1 \leq r<\infty$,

$$
\left\|A^{r}+B^{r}\right\| \leq\left\|(A+B)^{r}\right\|
$$

Lemma 4 ([10], Theorem 7) Suppose that two operators $A, B$ and positive real numbers $m, m^{\prime}, M, M^{\prime}$ satisfy either of the following conditions:

(1) $0<m \leq A \leq m^{\prime}<M^{\prime} \leq B \leq M$,

(2) $0<m \leq B \leq m^{\prime}<M^{\prime} \leq A \leq M$.

Then

$$
A \nabla_{\mu} B \geq K^{r}\left(h^{\prime}\right) A \sharp_{\mu} B
$$

for all $\mu \in[0,1]$, where $r=\min (\mu, 1-\mu)$ and $h^{\prime}=\frac{M^{\prime}}{m^{\prime}}$. 
Theorem 1 Let $0<m \leq A \leq m^{\prime}<M^{\prime} \leq B \leq M$. Then

$$
\frac{A+B}{2}+M m K^{\frac{1}{2}}\left(h^{\prime}\right)(A \sharp B)^{-1} \leq M+m,
$$

where $K\left(h^{\prime}\right)=\frac{\left(h^{\prime}+1\right)^{2}}{4 h^{\prime}}$ with $h^{\prime}=\frac{M^{\prime}}{m^{\prime}}$.

Proof It is easy to see that

$$
\frac{1}{2}(M-A)(m-A) A^{-1} \leq 0,
$$

then

$$
M m \frac{A^{-1}}{2}+\frac{A}{2} \leq \frac{M+m}{2} .
$$

Similarly,

$$
M m \frac{B^{-1}}{2}+\frac{B}{2} \leq \frac{M+m}{2} .
$$

Summing up the above two inequalities, we get

$$
\frac{A+B}{2}+M m \frac{A^{-1}+B^{-1}}{2} \leq M+m .
$$

By $(A \sharp B)^{-1}=A^{-1} \sharp B^{-1}$ and Lemma 4 , we have

$$
\begin{aligned}
\frac{A+B}{2}+M m K^{\frac{1}{2}}\left(h^{\prime}\right)(A \sharp B)^{-1} & =\frac{A+B}{2}+M m K^{\frac{1}{2}}\left(h^{\prime}\right)\left(A^{-1} \sharp B^{-1}\right) \\
& \leq \frac{A+B}{2}+M m \frac{A^{-1}+B^{-1}}{2} \\
& \leq M+m .
\end{aligned}
$$

This completes the proof.

Theorem 2 Let $0<m \leq A \leq m^{\prime}<M^{\prime} \leq B \leq M$. Then for every positive unital linear map $\Phi$,

$$
\Phi^{2}\left(\frac{A+B}{2}\right) \leq \frac{K^{2}(h)}{K\left(h^{\prime}\right)} \Phi^{2}(A \sharp B)
$$

and

$$
\Phi^{2}\left(\frac{A+B}{2}\right) \leq \frac{K^{2}(h)}{K\left(h^{\prime}\right)}(\Phi(A) \sharp \Phi(B))^{2},
$$

where $K(h)=\frac{(h+1)^{2}}{4 h}, K\left(h^{\prime}\right)=\frac{\left(h^{\prime}+1\right)^{2}}{4 h^{\prime}}, h=\frac{M}{m}$, and $h^{\prime}=\frac{M^{\prime}}{m^{\prime}}$. 
Proof The inequality (2.5) is equivalent to

$$
\left\|\Phi\left(\frac{A+B}{2}\right) \Phi^{-1}(A \sharp B)\right\| \leq \frac{K(h)}{K^{\frac{1}{2}}\left(h^{\prime}\right)} .
$$

Compute

$$
\begin{aligned}
\| & \Phi\left(\frac{A+B}{2}\right) M m K^{\frac{1}{2}}\left(h^{\prime}\right) \Phi^{-1}(A \sharp B) \| \\
& \leq \frac{1}{4}\left\|\Phi\left(\frac{A+B}{2}\right)+M m K^{\frac{1}{2}}\left(h^{\prime}\right) \Phi^{-1}(A \sharp B)\right\|^{2} \quad(\text { by }(2.1)) \\
& \left.\leq \frac{1}{4}\left\|\Phi\left(\frac{A+B}{2}\right)+M m K^{\frac{1}{2}}\left(h^{\prime}\right) \Phi\left((A \sharp B)^{-1}\right)\right\|^{2} \quad \text { (by }(2.2)\right) \\
& =\frac{1}{4}\left\|\Phi\left(\frac{A+B}{2}+M m K^{\frac{1}{2}}\left(h^{\prime}\right)(A \sharp B)^{-1}\right)\right\|^{2} \\
& \leq \frac{1}{4}\|\Phi(M+m)\|^{2} \quad(\text { by }(2.4)) \\
& =\frac{1}{4}(M+m)^{2} .
\end{aligned}
$$

That is,

$$
\left\|\Phi\left(\frac{A+B}{2}\right) \Phi^{-1}(A \sharp B)\right\| \leq \frac{(M+m)^{2}}{4 M m K^{\frac{1}{2}}\left(h^{\prime}\right)}=\frac{K(h)}{K^{\frac{1}{2}}\left(h^{\prime}\right)} .
$$

Thus, (2.7) holds. The proof of (2.6) is similar, we omit the details.

This completes the proof.

Remark 1 Because of $\frac{K^{2}(h)}{K\left(h^{\prime}\right)}<K^{2}(h)$, inequalities (2.5) and (2.6) are refinements of (1.1) and (1.2), respectively.

Theorem 3 Let $0<m \leq A \leq m^{\prime}<M^{\prime} \leq B \leq M$ and $2 \leq p<\infty$. Then for every positive unital linear map $\Phi$,

$$
\Phi^{2 p}\left(\frac{A+B}{2}\right) \leq \frac{1}{16}\left(\frac{K^{2}(h)\left(M^{2}+m^{2}\right)^{2}}{K\left(h^{\prime}\right) M^{2} m^{2}}\right)^{p} \Phi^{2 p}(A \sharp B)
$$

and

$$
\Phi^{2 p}\left(\frac{A+B}{2}\right) \leq \frac{1}{16}\left(\frac{K^{2}(h)\left(M^{2}+m^{2}\right)^{2}}{K\left(h^{\prime}\right) M^{2} m^{2}}\right)^{p}(\Phi(A) \sharp \Phi(B))^{2 p},
$$

where $K(h)=\frac{(h+1)^{2}}{4 h}, K\left(h^{\prime}\right)=\frac{\left(h^{\prime}+1\right)^{2}}{4 h^{\prime}}, h=\frac{M}{m}$, and $h^{\prime}=\frac{M^{\prime}}{m^{\prime}}$.

Proof By the operator reverse monotonicity of inequality (2.5), we have

$$
\Phi^{-2}(A \sharp B) \leq L^{2} \Phi^{-2}\left(\frac{A+B}{2}\right),
$$

where $L=\frac{K(h)}{K^{\frac{1}{2}}\left(h^{\prime}\right)}$. 


\section{Compute}

$$
\begin{aligned}
\| & \Phi^{p}\left(\frac{A+B}{2}\right) M^{p} m^{p} \Phi^{-p}(A \sharp B) \| \\
& \leq \frac{1}{4}\left\|L^{\frac{p}{2}} \Phi^{p}\left(\frac{A+B}{2}\right)+\left(\frac{M^{2} m^{2}}{L}\right)^{\frac{p}{2}} \Phi^{-p}(A \sharp B)\right\|^{2} \quad(\text { by }(2.1)) \\
& \leq \frac{1}{4}\left\|L \Phi^{2}\left(\frac{A+B}{2}\right)+\frac{M^{2} m^{2}}{L} \Phi^{-2}(A \sharp B)\right\|^{p} \quad(\text { by }(2.3)) \\
& \leq \frac{1}{4}\left\|L \Phi^{2}\left(\frac{A+B}{2}\right)+L M^{2} m^{2} \Phi^{-2}\left(\frac{A+B}{2}\right)\right\|^{p} \quad(\text { by }(2.10)) \\
& \leq \frac{1}{4}\left(L\left(M^{2}+m^{2}\right)\right)^{p} \quad(\text { by }[1],(4.7)) .
\end{aligned}
$$

That is,

$$
\left\|\Phi^{p}\left(\frac{A+B}{2}\right) \Phi^{-p}(A \sharp B)\right\| \leq \frac{1}{4}\left(\frac{L\left(M^{2}+m^{2}\right)}{M m}\right)^{p}=\frac{1}{4}\left(\frac{K^{2}(h)\left(M^{2}+m^{2}\right)^{2}}{K\left(h^{\prime}\right) M^{2} m^{2}}\right)^{\frac{p}{2}} .
$$

Thus, (2.8) holds. By inequality (2.6), the proof of (2.9) is similar, we omit the details.

This completes the proof.

Remark 2 Since $K\left(h^{\prime}\right)>1$, inequalities (2.8) and (2.9) are sharper than (1.3) and (1.4), respectively.

Theorem 4 Let $0<m \leq A \leq M$ and let $X, Y$ be two isometries on $\mathcal{H}$ whose final spaces are orthogonal to each other. Then for every 2-positive unital linear map $\Phi$,

$$
\left\|\Phi\left(X^{*} A Y\right) \Phi\left(Y^{*} A Y\right)^{-1} \Phi\left(Y^{*} A X\right) \Phi\left(X^{*} A X\right)^{-1}\right\| \leq \frac{(M-m)^{2}}{4 M m} .
$$

Proof Since $X$ is isometric and $0<m \leq A \leq M, m \leq \Phi\left(X^{*} A X\right) \leq M$ and $\frac{1}{M} \leq$ $\Phi\left(X^{*} A X\right)^{-1} \leq \frac{1}{m}$.

Compute

$$
\begin{aligned}
& \left(\frac{M-m}{M+m}\right)^{2} M m\left\|\Phi\left(X^{*} A Y\right) \Phi\left(Y^{*} A Y\right)^{-1} \Phi\left(Y^{*} A X\right) \Phi\left(X^{*} A X\right)^{-1}\right\| \\
& \quad \leq \frac{1}{4}\left\|\Phi\left(X^{*} A Y\right) \Phi\left(Y^{*} A Y\right)^{-1} \Phi\left(Y^{*} A X\right)+\left(\frac{M-m}{M+m}\right)^{2} M m \Phi\left(X^{*} A X\right)^{-1}\right\|^{2} \quad(\text { by }(2.1)) \\
& \left.\quad \leq \frac{1}{4}\left\|\left(\frac{M-m}{M+m}\right)^{2} \Phi\left(X^{*} A X\right)+\left(\frac{M-m}{M+m}\right)^{2} M m \Phi\left(X^{*} A X\right)^{-1}\right\|^{2} \quad \text { (by }(1.5)\right) \\
& \quad \leq \frac{1}{4}\left(\frac{M-m}{M+m}\right)^{4}(M+m)^{2}
\end{aligned}
$$

Hence,

$$
\left\|\Phi\left(X^{*} A Y\right) \Phi\left(Y^{*} A Y\right)^{-1} \Phi\left(Y^{*} A X\right) \Phi\left(X^{*} A X\right)^{-1}\right\| \leq \frac{(M-m)^{2}}{4 M m} .
$$

This completes the proof. 
Remark 3 Since $0<m \leq M$,

$$
\frac{1}{4}\left(\left(\frac{M-m}{M+m}\right)^{2} M+\frac{1}{m}\right)^{2} \geq\left(\frac{M-m}{M+m}\right)^{2} \frac{M}{m} \geq\left(\frac{M-m}{M+m}\right)^{2} \frac{(M+m)^{2}}{4 M m}=\frac{(M-m)^{2}}{4 M m}
$$

Thus, (2.11) is tighter than (1.6).

\section{Competing interests}

The authors declare that they have no competing interests.

\section{Authors' contributions}

All authors contributed equally to the writing of this paper. All authors read and approved the final manuscript.

\section{Author details}

${ }^{1}$ Oxbridge College, Kunming University of Science and Technology, Kunming, Yunnan 650106, P.R. China. ${ }^{2}$ Faculty of Science, Kunming University of Science and Technology, Kunming, Yunnan 650500, P.R. China.

\section{Acknowledgements}

The authors wish to express their heartfelt thanks to the referees for their detailed and helpful suggestions for revising the manuscript. This research was supported by Scientific Research Fund of Yunnan Provincial Education Department (No. 2014C206Y).

Received: 9 October 2015 Accepted: 15 January 2016 Published online: 27 January 2016

\section{References}

1. Lin, M: Squaring a reverse AM-GM inequality. Stud. Math. 215, 187-194 (2013)

2. Zhang, P: More operator inequalities for positive linear maps. Banach J. Math. Anal. 9, 166-172 (2015)

3. Kubo, F, Ando, T: Means of positive linear operators. Math. Ann. 246, 205-244 (1980)

4. Bhatia, R, Davis, C: More operator versions of the Schwarz inequality. Commun. Math. Phys. 215, $239-244$ (2000)

5. Lin, M: On an operator Kantorovich inequality for positive linear maps. J. Math. Anal. Appl. 402, 127-132 (2013)

6. Fu, X, He, C: Some operator inequalities for positive linear maps. Linear Multilinear Algebra 65, 571-577 (2015)

7. Bhatia, R, Kittaneh, F: Notes on matrix arithmetic-geometric mean inequalities. Linear Algebra Appl. 308, $203-211$ (2000)

8. Bhatia, R: Positive Definite Matrices. Princeton University Press, Princeton (2007)

9. Ando, T, Zhan, X: Norm inequalities related to operator monotone functions. Math. Ann. 315, 771-780 (1999)

10. Zuo, H, Shi, G, Fujii, M: Refined Young inequality with Kantorovich constant. J. Math. Inequal. 5, 551-556 (2011)

\section{Submit your manuscript to a SpringerOpen ${ }^{\circ}$ journal and benefit from:}

- Convenient online submission

Rigorous peer review

- Immediate publication on acceptance

Open access: articles freely available online

- High visibility within the field

- Retaining the copyright to your article 